Journal of Case Reports 2018;8(2):115-119

\title{
Spontaneous Muscle Hematoma in Dengue Hemorrhagic Fever: A Report of Four Cases
}

\author{
Bhargavan Pallivalappil, Supreeth Ramesh Naga, Dipu KP, Robin George Manappallil \\ Department of Internal Medicine, Baby Memorial Hospital, Calicut, Kerala, India.
}

\section{Corresponding Author: \\ Dr. Supreeth R N \\ Email: supreeth22@gmail.com}

This is an Open Access article distributed under the terms of the Creative Commons Attribution License (creativecommons.org/ licenses/by/3.0).

Received Accepted

Published

March 11, 2018

April 14, 2018

May 10, 2018

\begin{abstract}
Background: Dengue is the most common arboviral infection in the world. Dengue hemorrhagic fever (DHF) presents with manifestations like bleeding gums, melena, menorrhagia, epistaxis and hemoptysis which are well documented. Spontaneous intramuscular hematoma is a rare manifestation of DHF and very few such cases were reported so far. Case Report: We report four cases of DHF complicated by spontaneous muscle hematomas, of which three were managed conservatively and one case needed transcatheter embolization. Conclusion: Sudden onset abdominal or back pain with decreasing hemoglobin level in the setting of dengue fever should incite a suspicion of spontaneous muscle hematoma. Nevertheless, orificial bleeding should be ruled out first. Diagnosis is by ultrasonogram or computerised tomography. Such cases are managed conservatively with fluid resuscitation, packed cell transfusion and analgesia. Most of the patients improve well with appropriate management. Intervention by embolization is rarely needed.
\end{abstract}

Keywords: Abdominal Wall, Dengue, Epistaxis, Hematoma, Hemoptysis.

\section{Introduction}

Dengue fever is caused by a Flavivirus with 5 serotypes. It is one of the most rapidly emerging arboviral infections in India as well as globally. The global incidence of dengue is estimated to be around 400 million every year. The increasing trend in dengue cases can be attributed to the factors like urbanization, globalization and lack of effective mosquito control measures in developing countries [1]. Spontaneous muscle hematoma is seen usually in obesity, pregnancy, female gender, chronic cough and anticoagulation therapy [2]. Spontaneous muscle and muscle sheath hematoma is an infrequent consequence of DHF with serious clinical implications. With these four cases, we highlight the seriousness of this so far underreported manifestation and its management.

\section{Case Reports}

\section{Case 1}

A 53 year old female presented with high grade intermittent fever associated with chills, myalgia and multiple episodes of non-bilious, nonprojectile vomiting of three days duration. On examination, she had pulse rate of $115 / \mathrm{min}$, blood pressure $130 / 80 \mathrm{~mm} \mathrm{Hg}$, respiratory rate $30 / \mathrm{min}$, temperature $100^{\circ} \mathrm{F}$. Systemic examination was normal. Blood tests showed hemoglobin: $12.6 \mathrm{~g} / \mathrm{dL}$, red blood cell count: $4,28,000 / \mathrm{mm}^{3}$, total leukocyte count: $3,200 / \mathrm{mm}^{3}$, packed cell volume: $44 \%$ and platelet count: $1,10,000 / \mathrm{mm}^{3}$. Her serum bilirubin was $1.3 \mathrm{mg} / \mathrm{dL}$, aspartate transaminase (AST) was $60 \mathrm{U} / \mathrm{L}$, alanine transaminase (ALT) was $39 \mathrm{U} / \mathrm{L}$ and alkaline phosphatase (ALP) was $108 \mathrm{U} / \mathrm{L}$. Dengue NS1 antigen assay by rapid diagnostic 
test (RDT) and dengue IgM ELISA were positive. Other blood parameters were normal. On day 5, she developed sudden onset left sided abdominal pain and examination revealed round tender mass in the left para-umbilical area. Blood test showed sudden drop in hemoglobin to $6.5 \mathrm{~g} / \mathrm{dL}, \mathrm{RBC}$ count $2,20,000 / \mathrm{mm}^{3}$, PCV $26 \%$ and platelet count $28,000 / \mathrm{mm}^{3}$. Her prothrombin time (PT) was 10.5 sec, international normalised ratio (INR) 1.14 and activated partial thromboplastin time (aPTT) 26 sec. CT abdomen showed findings suggestive of left rectus sheath hematoma (RSH) and hematoma in the right psoas muscle [Fig.1].

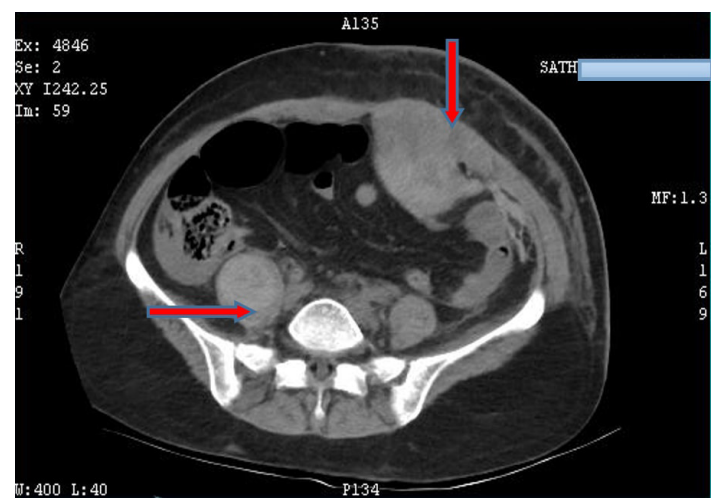

Fig.1: Axial section of CT abdomen showing hyperdense lesions in left rectus abdominis and right psoas muscles suggestive of muscular hematomas.

\section{Case 2}

A 67 year old house-wife came with high grade intermittent fever, tiredness, nausea, non-bilious vomiting of 5 days duration and left sided abdominal pain of 2 days duration. She is a hypertensive, on atenolol for past 10 years. On examination, her BP was $90 / 60 \mathrm{mmHg}$, pulse $62 / \mathrm{min}$, respiratory rate $22 / \mathrm{min}$ and temperature $99^{\circ} \mathrm{F}$. She had a large ecchymotic patch over the left postero-lateral chest wall and abdominal wall [Fig.2]. Abdominal examination revealed guarding and tenderness in the left iliac fossa. Other systems were normal. Blood tests showed hemoglobin: $5.3 \mathrm{~g} / \mathrm{dL}$, red blood cell count: $2,22,000 / \mathrm{mm}^{3}$, total leukocyte count: $9,300 / \mathrm{mm}^{3}$, platelet count: $76,000 / \mathrm{mm}^{3}$ and packed cell volume: $23 \%$. PT was 11.3 seconds, INR was 0.98 and aPTT was
27.8 seconds. Dengue NS1 antigen by RDT and dengue IgM antibody by ELISA were positive. In view of abdominal pain and anemia, contrast enhanced computed tomography (CECT) abdomen was obtained with a suspicion of intra-abdominal hemorrhage. CT showed large hematoma involving the subcutaneous, inter and intra-muscular planes in the postero-lateral aspect of left lower chest wall, extending to antero-lateral abdominal wall and small hematoma involving right lower abdominal wall (oblique muscles) [Fig.3,4].

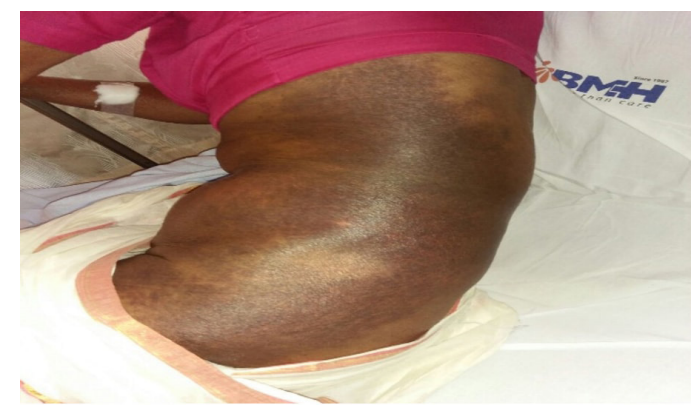

Fig.2: Large ecchymotic patch over the left postero-lateral thoracic and abdominal wall.

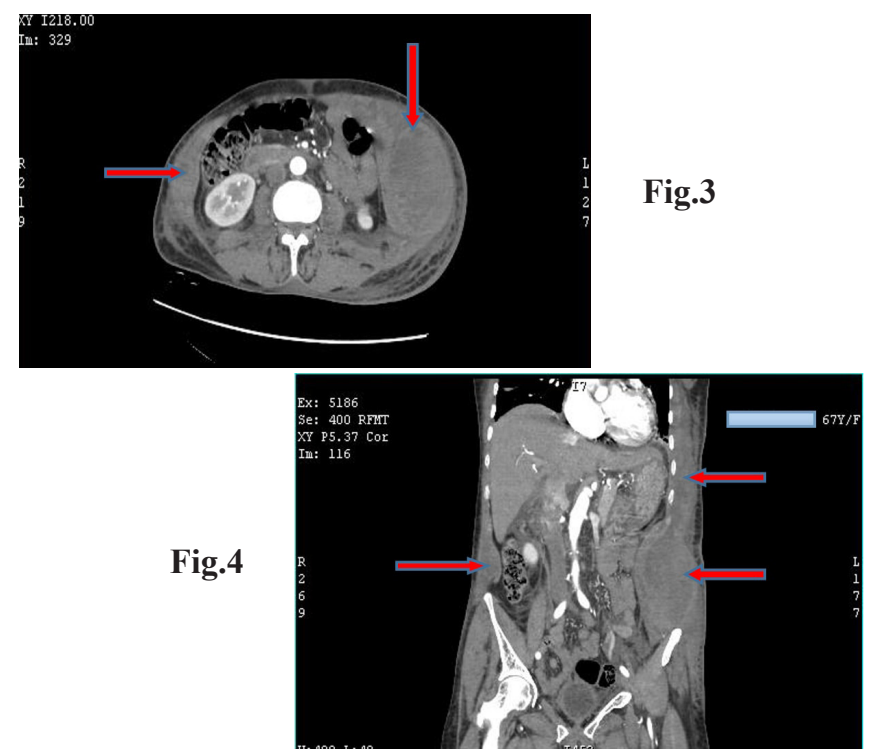

Fig.3,4: Axial and coronal sections of CECT abdomen showing large hematoma involving the subcutaneous, inter and intramuscular planes in the postero-lateral aspect of left lower chest wall, extending to antero-lateral abdominal wall with maximum thickness in the left lumbar and iliac fossa regions. Small hematoma involving right lower abdominal wall in the oblique muscles is seen. 


\section{Case 3}

A 65 year old male came to medicine outpatient department with high grade, intermittent fever, myalgia of 5 days duration and nausea with vomiting of 2 days duration. He gave a history of right renal calculus for the past 10 years and he took Ayurvedic treatment. On examination, his blood pressure was $100 / 80 \mathrm{~mm} \mathrm{Hg}$, pulse $66 / \mathrm{min}$, respiratory rate 34/ min and temperature $98.4^{\circ} \mathrm{F}$. Systemic examination was normal. Blood tests showed hemoglobin: $13 \mathrm{~g} /$ dL, red blood cell count: $4,30,000 / \mathrm{mm}^{3}$, packed cell volume: $38.8 \%$, total leukocyte count: $3,100 / \mathrm{mm}^{3}$ and platelet count: $28,000 / \mathrm{mm}^{3}$. Liver function test showed bilirubin: $2 \mathrm{mg} / \mathrm{dL}$, AST: $54 \mathrm{U} / \mathrm{L}$, ALT 33 $\mathrm{U} / \mathrm{L}$ and ALP of $38 \mathrm{U} / \mathrm{L}$. Other blood tests were normal. He tested positive for dengue NS1 antigen by rapid diagnostic kit test and IgM antibody by ELISA method. By day two, his platelet count dropped to $10,000 / \mathrm{mm}^{3}$ and he was given platelet transfusion, following which the count raised to $13,000 / \mathrm{mm}^{3}$. On day four, he developed acute onset right sided abdominal pain and on examination he had tenderness over right lumbar area without any palpable mass. Blood test showed drop in hemoglobin: $8.5 \mathrm{~g} / \mathrm{dL}, \mathrm{RBC}$ count $3,11,000 / \mathrm{mm}^{3}$, packed cell volume: $25.4 \%$ and platelet count: $42,000 / \mathrm{mm}^{3}$. PT was 11.7 seconds, INR 1.07 and aPTT 27.6 seconds. Ultrasound (USG) abdomen showed right psoas hematoma [Fig.5].

All the three patients were treated with intravenous fluids, platelet, packed cell, fresh frozen plasma transfusion and adequate analgesia. They improved well and were symptom free on review. Repeat ultrasound scan showed reduction in the size of hematoma.

\section{Case 4}

A 64 year old male retired employee came with history of high grade fever 10 days back that subsided within 3 days. He was treated at a primary centre in his village as dengue fever, from which he recovered in 4 days. Two days later, he developed constipation, nausea, fatigability and abdominal

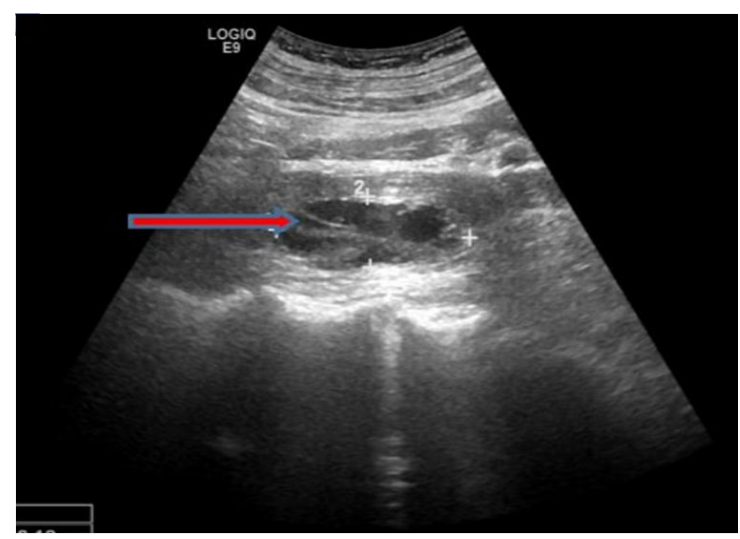

Fig.5: Ultrasound abdomen showing well defined fluid collection measuring $61 \times 24 \times 20 \mathrm{~mm}$ with echogenic particles and multiple strands in right psoas muscle suggestive of intramuscular hematoma.

pain with progressive abdominal distension. His blood pressure on admission was $130 / 80 \mathrm{mmHg}$, pulse: $88 /$ minute, respiratory rate: $23 /$ minute and temperature: $99^{\circ} \mathrm{F}$. Abdomen was tense and distended with tenderness at left hypochondric, lumbar and iliac fossa areas. Bowel sounds were absent. Blood tests showed hemoglobin: $7.9 \mathrm{~g} /$ dL, total leukocyte count: $18,000 / \mathrm{mm}^{3}$ with $88 \%$ neutrophils, platelet count: $81,000 / \mathrm{mm}^{3}$, PCV: 23\%, AST: $73 \mathrm{U} / \mathrm{L}$, ALT: $72 \mathrm{U} / \mathrm{L}$ and ALP was 79 U/L. Serum sodium was $119 \mathrm{mEq} / \mathrm{L}$. Dengue IgM antibody was positive by ELISA method. PT was 10.7 seconds, aPTT 21.5 seconds and INR 0.95. We considered possibilities of paralytic ileus, intestinal perforation and peritonitis clinically. CECT abdomen showed large retroperitoneal hematoma on left side extending into left psoas muscle with evidence of active contrast extravasation. There was no evidence of dynamic intestinal obstruction [Fig.6]. Repeat blood test after 2 hours showed hemoglobin drop to $6.9 \mathrm{~g} / \mathrm{dL}$. He was shifted to cath lab for endovascular embolization under general anaesthesia. Angiogram revealed three bleeding intercostal arteries on left side which were embolized with gel foam and hemostasis was achieved. He was treated with packed cell, platelet, fresh frozen plasma transfusion, analgesia and fluid therapy. Intravenous antibiotics were also administered in view of polymorphonuclear 
leucocytosis. He recovered after 7 days and repeat ultrasound showed reduction in the size of hematoma. He was better on follow up, 7 days after discharge.

\section{Discussion}

DHF is characterized by various hemorrhagic manifestations ranging from ecchymosis to gastrointestinal bleed, capillary leak, hypotension and shock. Spontaneous muscle hematoma is an uncommon cause of acute abdominal pain and rare manifestation of DHF. The factors influencing the severity of hemorrhagic manifestations in dengue are the host factors - antibody dependent enhancement (ADE) of viral replication \& the viral factors - variation in the virulence of various dengue viral strains [3]. Pathophysiology of hemorrhage in DHF is poorly understood even now. It is postulated that a combination of vasculopathy, coagulopathy and thrombopathy could be the probable mechanism. Vasculopathy is caused by TNF, IL-6 \& IL-8 mediated damage to the endothelial layer exposing the subendothelial von Willebrand (vWF) that leads to platelet aggregation [4]. In a prospective study reported by Wills et al. in 2002, 167 Vietnamese children showed minor prolongation of PT and APTT, as in our patients. Moderate decrease in fibrinogen level was also noted. Out of 167, 48 children showed low plasma concentrations of anticoagulant protein C, S and antithrombin III due to capillary leak [5]. There was concurrent increase in thrombomodulin, tissue factor and plasminogen activation inhibitor-1 (PAI-1) due to increased production following endothelial activation [5,6]. Virus-antibody complexes and infected macrophages activate the coagulation cascade followed by the activation of fibrinolytic system that results in production of D-dimer. Fibrinogen, factors II, V, VII, VIII, IX and $\mathrm{X}$ and plasminogen levels decrease leading to coagulopathy [4]. Thrombocytopenia is caused by the decreased production, increased destruction and aggregation of platelets due to vasculopathy. Elevated prostacyclin (PGI2) to thromboxane A2

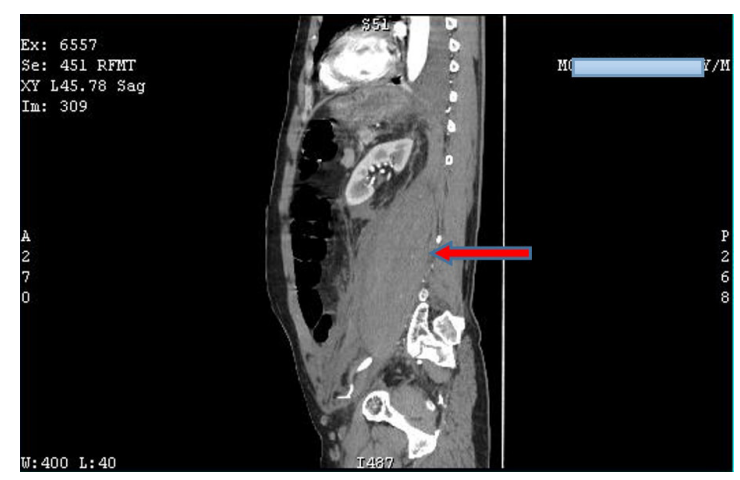

Fig.6: Sagittal section of CECT abdomen showing a large hematoma extending from D12 to L4 vertebra, pushing the left kidney anteriorly. The hematoma is extending upto left psoas muscle.

ratio leads to survival of dysfunctional platelets [7]. This could be one of the contributing factors of hematoma formation inspite of timely platelet transfusion in third case of our series. The mechanism of coagulopathy varies from classical disseminated intravascular coagulation (DIC) due to intact protein $\mathrm{C}$ pathway and increased thrombomodulin level $[5,8]$. Detailed coagulation workup was not done in our patients due to financial constraints.

Ultrasound and CECT are the diagnostic methods of choice for muscle hematomas. Most of the patients improve with adequate fluid resuscitation, platelet transfusion, packed cell transfusion and analgesia. Cases of active bleeding are seldom seen and they are managed with endovascular embolization.

\section{Conclusion}

Inter-muscular and intra-muscular hematoma, though uncommon, should always be considered in any proven dengue patient with acute pain. Capillary leak and old age are the risk factors. It is crucial to monitor hemoglobin, hematocrit and platelet count, daily, as it is difficult to diagnose this condition clinically. Sudden or progressive decline in hemoglobin and hematocrit levels without any external evidence of bleeding and appearance of painful swelling at unusual site should prompt a 


\section{clinician to evaluate for occult hemorrhage through} relevant imaging studies.

Acknowledgement: We thank Dr. Sana Sharfin Bisma and Dr. Abin Ummer, Department of Radiology, Baby Memorial Hospital for helping us with interpretation of imaging studies. Contributors: PVB: patient management, literature search, critical evaluation of manuscript and manuscript editing, RNS: manuscript writing, literature search and assisted PVB in patient management, KPD, RGM: patient management and key inputs into manuscript. RNS will act as the guarantor. All authors approved the final version of this manuscript.

Funding: None; Competing interests: None stated.

\section{References}

1. Wilder-Smith A, Murray, Quam M. Epidemiology of dengue: past, present and future prospects. Clin Epidemiol. 2013;5:299-309.

2. Eckhoff K, Wedel T, Both M, Bas K, Maass N, Alkatout I. Spontaneous rectussheath hematoma in pregnancy and a systematic anatomical workup of rectus sheath hematoma: a case report. J Med Case Rep. 2016;10:292.
3. Bäck AT, Lundkvist $\AA$. Dengue viruses - an overview. Infect Ecol \& Epidemiol. 2013;3:19839.

4. Suseno A, Nasronudin N. Pathogenesis of hemorrhagic due to dengue virus. Indonesian Journal of Tropical and Infectious Disease. 2015;5:107.

5. Wills B, Oragui E, Stephens A, Daramola O, Dung N, Loan $\mathrm{H}$, et al. Coagulation abnormalities in dengue hemorrhagic fever: serial investigations in 167 Vietnamese children with dengue shock syndrome. Clin Infect Dis. 2002;35:277-285.

6. Anderson R, Wang S, Osiowy C, Issekutz A. Activation of endothelial cells via antibody-enhanced dengue virus infection of peripheral blood monocytes. J Virol. 1997;71:4226-4232.

7. Preeyasombat C, Treepongkaruna S, Sriphrapradang A, Choubtum L. The role of prostacyclin (PGI2) and thromboxane A2 (TXA2) in pathogenesis of dengue hemorrhagic fever (DHF). J Med Assoc of Thai. 1999;82 Suppl 1:S16-21.

8. Faust SN, Levin M, Harrison OB, Goldin RD, Lockhart MS, Kondaveeti S, et al. Dysfunction of the endothelial protein $\mathrm{C}$ activation pathway in severe meningococcal sepsis. N Engl J Med. 2001;345:408-416. 